\title{
SIMPLE PDE MODEL OF SPOT REPLICATION IN ANY DIMENSION
}

\author{
CHIUN-CHUAN CHEN AND THEODORE KOLOKOLNIKOV
}

\begin{abstract}
We propose a simple PDE model which exhibits self-replication of spot solutions in any dimension. This model is analysed in one and higher dimensions. In one dimension, we rigorously demonstrate that the conditions proposed by Nishiura and Ueyama for self-replication are satisfied. In dimension three, two different types of replication mechanisms are analysed. The first type is due to radially symmetric instability, whereby a spot bifurcates into a ring. The second type is non-radial instability, which causes a spot to deform into a peanut-like shape, and eventually split into two spots. Both types of replication are observed in our model, depending on parameter choice. Numerical simulations are shown confirming our analytical results.
\end{abstract}

\section{INTRODUCTION}

In this paper we present a simple non-autonomous PDE which exhibits the self-replication of a spot solution in $\mathbb{R}^{N}, N \geq 1$. The PDE is

$$
u_{t}=\Delta u-u+\frac{\left(1+a|x|^{q}\right) u^{p}}{\int_{\mathbb{R}^{N}}\left(1+a|x|^{q}\right) u^{p+1}}, \quad x \in \mathbb{R}^{N} ; \quad \nabla u(0, t)=0
$$

Examples of this phenomenon are shown in Figure 1. Self-replication was first observed by Pearson in the Gray-Scott model [23]. Since then, many theoretical and numerical studies have looked at self-replication in both one and two spatial dimensions for the Gray-Scott model in different parameter regimes $([25],[24],[21],[22],[19],[3],[2],[14],[4])$. Many other reaction-diffusion systems have been found to exhibit self-replication behaviour. These include the ferrocyanideiodide-sulfite system ([11]), the Belousov-Zhabotinsky reaction ([12], [18] ), the Gierer-Meinhardt model ([16], [9], [15]), the Bonhoffer van-der-Pol-type system ([6], [7]) and the Brusselator [13].

In an effort to classify reaction-diffusion systems that can exhibit pulse self-replication, Nishiura and Ueyema, motivated by the numerical study of the Gray-Scott model, proposed a set of necessary conditions for this phenomenon to occur in [21]. Roughly stated, these conditions are the following:

The disappearance of the ground-state solution due to a fold point (saddle-node bifurcation) that occurs when a control parameter is increased above a certain threshold value. The existence of a dimple eigenfunction at the fold point, which is believed to be responsible for the initiation of the self-replication process. By definition, a dimple eigenfunction is a radially symmetric eigenfunction $\Phi(|x|)$ associated with a zero eigenvalue at the fold point, that decays as $|x| \rightarrow \infty$ and that has a positive zero (See figure 3 ).

Stability of the steady-state solution on one side of the fold point.

The alignment of the fold points, so that the disappearance of $K$ ground states, with $K=1,2,3, \ldots$, occurs at asymptotically the same value of the control parameter. 


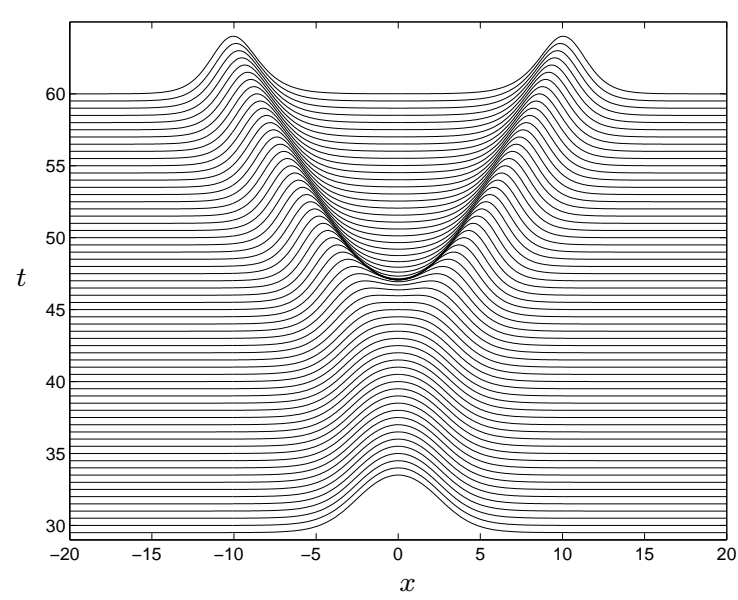

(a)
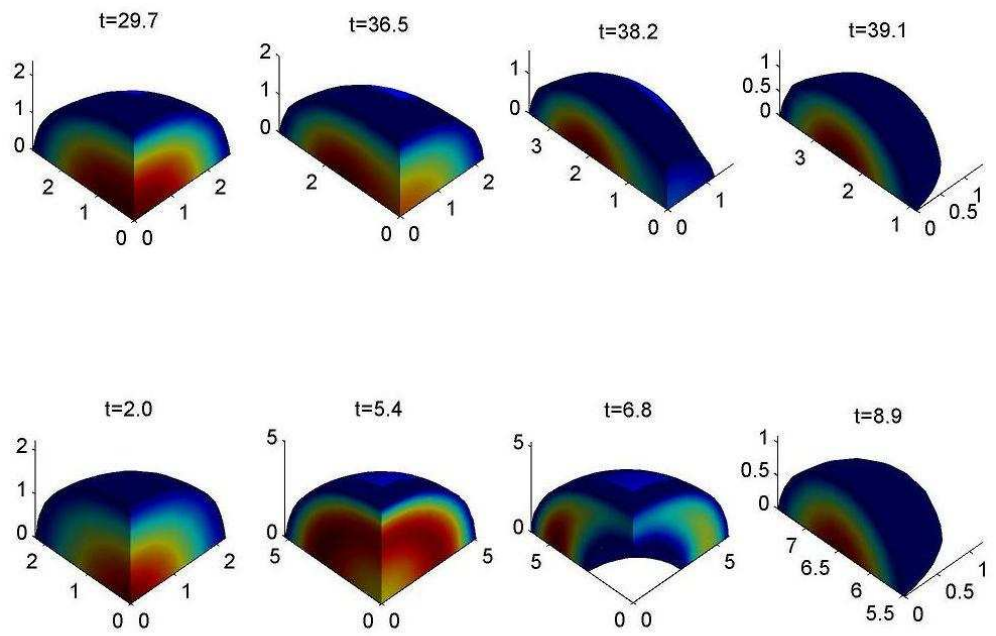

(b)

FIGURE 1. (a): Numerical simulation of (1) in one dimension with $p=2, q=2, a=0.08$. Selfreplication is observed. (b): Numerical simulation of (1) in three dimensions, showing two different types of self-replication. The snapshots show the cross-section of the solution in the first quadrant $x, y, z>0$. The surface corresponds to the contour $u=0.6 \max (u)$; cross-sections $x=0$ and $y=0$ are shown in using colormap (online) with red corresponding to $\max (u)$ and blue to $0.6 \max (u)$. First row: Spot-to-spot bifurcation due to instability non-radial eigenfunction. The parameters are $p=2, q=1.3$ and $a=0.5$. Second row: spot-to-ring bifurcation due to radial instability. The parameters are: $p=2, q=3, a=0.035$. The spot-to-ring bifurcation is followed by ring-to-spots bifurcation.

Conditions (S1) (S2) and (S3) are sufficient for self-replication to occur; condition S4 is needed for a cascade of self-replication to take place but is not necessary for a single self-replication event. These conditions were first verified numerically for a certain regime of the Gray-Scott model in [21], [5]. In a different regime, the Gray-Scott model reduces to the so-called core problem [19], [4], [14]. After a scaling, the core problem is

$$
\left\{\begin{array}{c}
U_{r r}+\frac{N-1}{r} U_{r}-U+U^{2} V=0 ; \quad V_{r r}+\frac{N-1}{r} V_{r}-a U^{2} V=0 \\
V(0)=1 ; \quad V^{\prime}(0)=0=U^{\prime}(0) \\
V, U>0 ; \quad U \rightarrow 0 \text { as } r \rightarrow \infty
\end{array}\right.
$$

The existence of a fold point of (2) in one dimension was shown numerically in [19]. This was proven analytically in [4]. On the other hand, conditions S2 and S3 were verified only numerically for (2); up to this day it is an open question to verify them analytically. There are few analytical results for (2) in two or three dimensions (but see [19] for some partial results).

In this paper we show analytically that the simple model (1) can exhibit self-replication in any dimension, for some parameter values of $p, q$ as $a$ is sufficiently increased from zero. We analytically 
verify conditions S1 to S3. In particular, we show that these conditions hold provided that

$$
\begin{gathered}
p>1 \text { and } q>\frac{(p-1) N}{2} \text { if } N=1 \text { or } 2 \\
1<p<\frac{N+2}{N-2} \text { and } q>\frac{(p-1)(N-1)}{2} \text { if } N \geq 3 .
\end{gathered}
$$

Under these conditions, self-replication in (1) will occur as the parameter $a$ is increased past some critical value $a_{c}$. In one dimension, the bifurcation structure and the self-replication mechanism is analogous to what has been observed for the reduced Gray-Scott model (2); but unlike the studies [19], [4], we are able to verify not only condition S1 but also conditions S2 and S3 analytically.

In dimensions two and three, the self-replication conditions (S1-S3) leads to a radially symmetric bifurcation, whereby a spot bifurcates into a ring that concentrates on the surface of $N$-dimensional ball. However there is another self-replication mechanism that can occur. Namely, a spot can become unstable with respect to non-radial perturbations of mode 2. Numerically, this leads to what we shall call peanut splitting, whereby a radially symmetric spot starts to aquire a peanut-like shape, which eventually pinches off and becomes two spots. We study both types of self-replication of (1) in three dimensions; we demonstrate that both are possible depending on choice of parameters (see Figure 1(b)). Analytically, we show that when $N=3, p=2$ and $q=1$, the spot will undergo peanut splitting if $a$ is sufficiently large; whereas no spot-to-ring bifurcation is expected for any value of $a$. On the other hand, if $p=2, q>1$, both radial and nonradial splitting is possible. For $q$ sufficiently large, the radial splitting dominates as illustrated in Figure 1(b) row 1 . To the best of our knowledge, this is the first rigorous demonstration of self-replication in three dimensions.

The summary of the paper is as follows. In $\S 2$ we study the steady state problem associated with (1). The main result is Theorem 1, which proves the boundedness of the bifurcation diagram under assumptions (3), thus showing the existence of the fold point and verifying condition (S1). In $\S 3.1$ we study radial stability, and analytically verify conditions (S2) and (S3). This fully characterizes self-replication in one dimension, and also characterizes radial replication in dimensions $>1$. In $\S 3.2$ we address non-radial instability to complete the classification of self-replication phenomena in three dimensions. In $\S 4$ we discuss some generalizations, compare to other models with self-replication, provide some open problems and conlcuding remarks.

\section{Analysis of the Ground state.}

We start our analysis by considering the radially symmetric ground state solution of (1). After a scaling, the ground state solution satisfies satisfies

$$
u_{r r}+\frac{N-1}{r} u_{r}-u+u^{p}\left(1+a r^{q}\right)=0, \quad u^{\prime}(0)=0, \quad u \rightarrow 0 \text { as } r \rightarrow \infty, \quad u>0 .
$$

It is well known that the steady state problem (4) with $a=0$ admits a unique solution when $p \in\left(1, p^{*}\right)$ where

$$
p^{*}=\left\{\begin{array}{c}
(N+2) /(N-2), \quad N \geq 3 \\
\infty, \quad N \leq 2
\end{array}\right.
$$




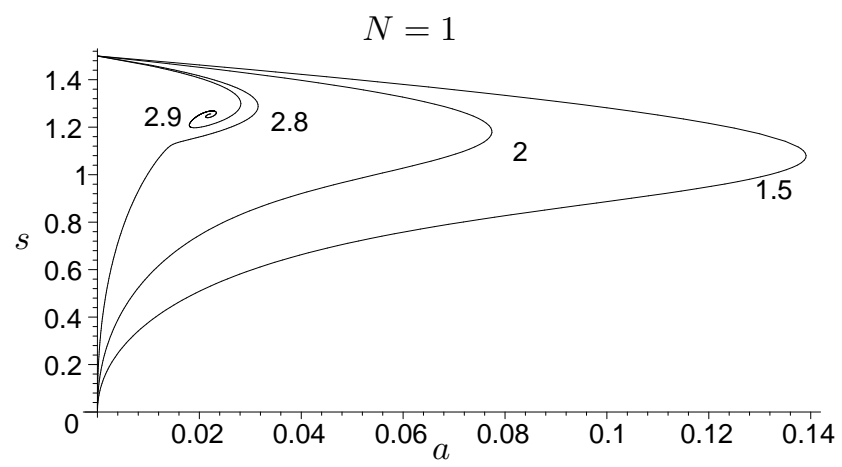

(a)

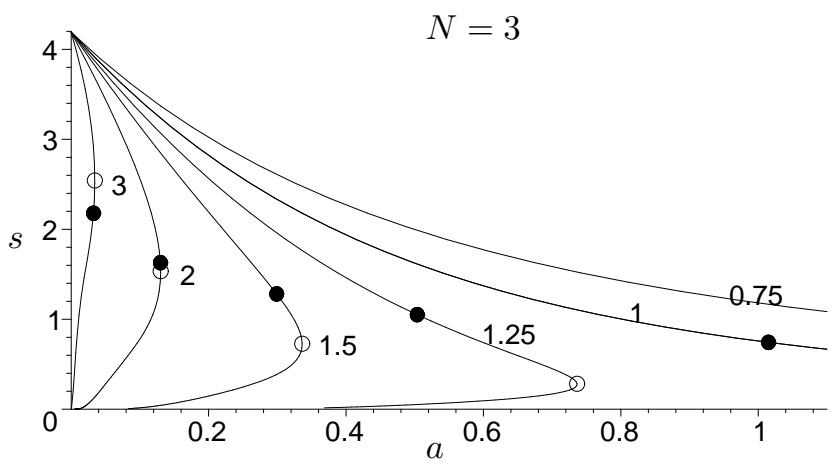

(b)

FiguRE 2. Bifurcation diagram for (4) of $a$ vs. $s=u(0)$ with $p=2$ and for several different values of $q$ as indicated. (a) $N=1$. There is a fold point for all values of $q$. The bifurcation graph changes its topology at around $q=2.8$, but is bounded for all $q$. (b) $N=3$. Fold point is indicated by an empty circle. Nonradial instability threshold is indicated with filled circle. If $q>2.1$ then fold-point instability dominates. If $q<2.1$ then non-radial instability dominates. The fold point exists if $q>1$; the bifurcation graph is unbounded if $q<1$.

is the critical exponent [1], [17]. However in general the solution is not unique when $a \neq 0$. As an example, consider (4) with $N=3, p=2$. The bifurcation diagram $s=u(0)$ vs. $a$ is computed numerically on Figure 2(b) for several different values of $q$. When $q>1$, the bifurcation curve is bounded and there is a fold point at some $a=a_{c}$ beyond which there are no solutions. This fold point is precisely Condition S1 which we wish to prove. On the other hand, if $q \leq 1$ then a solution exists for all $a>0$ with $s \rightarrow 0$ as $a \rightarrow \infty$. The main goal of this section is to classify under which conditions on $p, q, N$ the bifurcation graph is bounded in the $(a, s)$ plane - and therefore exhibits a fold point. The following theorem provides these bounds.

Theorem 1. Given $a \geq 0$, let $u(r)$ be a solution to (4) and let

$$
s:=u(0) .
$$

Define

$$
\begin{aligned}
& q_{\star}:=\frac{N(p-1)-2(p+1)}{2} ; \quad q^{\star}:=\frac{(p-1) N}{2} ; \\
& q_{c}:=\frac{(p-1)(N-1)}{2} .
\end{aligned}
$$

The following holds.

(i) Suppose that $p \in\left(1, p^{\star}\right)$ where $p^{\star}$ is the critical exponent given by (5) and $q \geq 0$. Given any constant $a_{0}>0$, there exists a constant $s_{0}=s_{0}\left(a_{0}, p, q\right)$ such that $s<s_{0}$ whenever $a \leq a_{0}$.

(ii) Suppose that either $N \geq 3$ and $q>q_{c}$ or else $N \leq 2$ and $q>q^{\star}$. There exists a constant $a_{0}$ such that $a<a_{0}$.

(iii) If $N \geq 3$ and $q_{\star}<q<q_{c}$ and $q \geq 0$ then the solution to (4) exists for all $a \geq 0$, provided that $1<p<p^{\star}$. 
When (i) and (ii) simultaneously hold, the bifurcation graph in the positive $(a, s)$ plane is bounded. Note that that $q_{\star}<0$ iff $p<p_{\star}$ and moreover $q_{\star}<q_{c}<q^{\star}$. In particular, statements (i), (ii) hold simultaneously in dimension $N \geq 3$ provided that $q>q_{c}$ and $p \in\left(1, p^{\star}\right)$; they hold in dimension $N=1$ or 2 provided that $q>q^{\star}$ and $p>1$. In conclusion, the bifurcation curve is guaranteed to have a fold point whenever (3) is satisfied, which proves the key condition (S1) for self-replication. This is in agreement with numerics as shown on Figure 2(a), where $N=3, p=2<p^{\star}=5$ : indeed the bifurcation curve is bounded and the fold point is observed whenever $q>1=q_{c}$. On the other hand the bifurcation curve is unbounded when $q \leq 1$; this is in agreement with statement (iv) of Theorem 1.

Remark 1. We think that $q^{\star}$ in (ii) can be replaced by $q_{c}$ and the condition $N \geq 3$ can be eliminated in (ii). However we were unable to prove that.

Remark 2. We also conjecture that the condition $p<p^{\star}$ is not necessary in (iii); it is sufficient that $q_{\star}<q<q_{c}$ for (iii) to hold.

The proof of (ii) and (iii) of Theorem 1 is an immediate consequence of the following lemma.

Lemma 2. Consider the problem

$$
u^{\prime \prime}+\frac{N-1}{r} u^{\prime}-u+\left(\varepsilon+r^{q}\right) u^{p}=0 ; \quad u^{\prime}(0)=0, \quad u>0 ; u \rightarrow 0 \quad \text { as } \quad r \rightarrow \infty .
$$

Suppose that $1<p<p^{\star}$, and let $q_{\star}, q^{\star}, q_{c}$ be as given by (7). We have the follows results.

(i) Suppose that $q$ satisfies

$$
q>q_{c} \text { if } N \geq 3 \text { or } q>q^{\star} \text { if } N \leq 2 .
$$

Then there exists $\varepsilon_{0}=\varepsilon_{0}(p, q, N)$ such that (8) has no solution for all $0 \leq \varepsilon<\varepsilon_{0}$.

(ii) Supopse that $N \geq 3$ and $q=q_{c}$ and $\varepsilon=0$. Then (8) has no solution.

(iii) Suppose that $N \geq 3$ and $q_{\star}<q<q_{c}$. Then the solution to (8) exists for all $\varepsilon>0$. Such solution is unique if $\varepsilon=0$.

We now give proofs of Theorem 1 and Lemma 2.

Proof of Theorem 1. We first show (i). Consider the initial value problem

$$
v_{r r}+\frac{N-1}{r} v_{r}-v+\left(1+a r^{q}\right) v^{p}=0, \quad v^{\prime}(0)=0, \quad v(0)=s .
$$

Rescale

$$
v=s V ; \quad r=\tau y .
$$

where $\tau$ is to be specified. Then the equation for $V$ is

$$
V_{y y}+\frac{N-1}{y} V_{y}-\tau^{2} V+\left(\tau^{2} s^{p-1}+a \tau^{q+2} s^{p-1} y^{q}\right) V^{p}=0 ; \quad V^{\prime}(0)=0, \quad V(0)=1 .
$$

Choosing $\tau=s^{-(p-1) / 2}$, we then obtain

$$
V_{y y}+\frac{N-1}{y} V_{y}+V^{p}-\varepsilon_{1} V+\varepsilon_{2} y^{q} V^{p}=0 ; \quad V^{\prime}(0)=0, \quad V(0)=1 .
$$

where

$$
\varepsilon_{1}=s^{-1 /(p-1) ;} \varepsilon_{2}=a s^{-q /(2 p-2)} .
$$


Now consider the limiting problem

$$
V_{0 y y}+\frac{N-1}{y} V_{0 y}+V_{0}^{p}=0 ; \quad V_{0}(0)=1, V_{0}^{\prime}(0)=0 .
$$

In Lemma 8 (see Appendix $\mathrm{C}$ ) we show that for $p \in\left(1, p^{\star}\right), V_{0}$ becomes negative at some $y=y_{0}$. In particular, there exists $y_{1}>y_{0}$ and $C_{1}>0$ such that $v_{0}\left(y_{1}\right)<-C_{1}<0$. By continuity of solutions to initial value problem with respect to parameters, $V$ can be made arbitrary close to $V_{0}$ by choosing any sufficiently small $\varepsilon_{1}, \varepsilon_{2}$. In particular, there exists a $\varepsilon=\varepsilon(p, q)>0$ such that for all $\varepsilon_{1}, \varepsilon_{2}<\varepsilon$, we have $\left|V\left(y_{1}\right)-V_{0}\left(y_{1}\right)\right|<C_{1} / 2 \Longrightarrow V\left(y_{1}\right)<0$. Now given $a_{0}>0$ and for any $0<a<a_{0}$, note that $\varepsilon_{1}, \varepsilon_{2}<\varepsilon$ whenever $s>s_{0}$, where $s_{0}:=\max \left(\varepsilon^{-(p-1)},\left(\varepsilon / a_{0}\right)^{-2(p-1) / q}\right)$. In this case, $v$ has a root and hence no solution to (4) exists when $a<1$ and $s>c_{1}$. This proves (i).

To prove (ii) we apply Lemma 2 after a change of variables $u \rightarrow a^{1 /(1-p)} u$. Then (4) becomes (8) with $\varepsilon=1 / a$. Statement (i) of Lemma 2 immediately yields the desired result. The proof of (iii) follows from statement (iii) of Lemma 2 .

Proof. of Lemma 2. We start with the nonexistence results (i) and (ii) which are proven in Steps 1 to 4. Result (iii) is proven in Step 5.

Step 1. We first derive the following key identity:

$$
\int_{0}^{\infty} r^{N-1} u^{p+1}\left[\varepsilon-c_{1} r^{q}\right]>0
$$

where

$$
c_{1}=\left\{\begin{array}{c}
\frac{2}{p+1}\left(q-q_{c}\right), \quad N \geq 3 \\
\frac{2}{(p+1) N}\left(q-q^{\star}\right), \quad N \leq 2 .
\end{array}\right.
$$

In one and two dimensions, this is a consequence of Pohozhaev-type inequalities as we now show. First, multiply (8) by $r^{N-1} u$ and integrate by parts to obtain

$$
-\int_{0}^{\infty} r^{N-1} u^{\prime 2}-\int_{0}^{\infty} r^{N-1} u^{2}+\int_{0}^{\infty} r^{N-1}\left(\varepsilon+r^{q}\right) u^{p+1}=0
$$

Next, multiply (8) by $r^{N} u^{\prime}$ and integrating by parts to get:

$$
\left(-1+\frac{N}{2}\right) \int_{0}^{\infty} r^{N-1} u^{\prime 2}+\frac{N}{2} \int_{0}^{\infty} r^{N-1} u^{2}-\frac{N+q}{p+1} \int_{0}^{\infty} r^{N-1+q} u^{p+1}-\varepsilon \frac{N}{p+1} \int_{0}^{\infty} r^{N-1} u^{p+1}=0 .
$$

Combining (16) and (17) we obtain

$$
\int_{0}^{\infty} r^{N-1} u^{p+1}\left[\varepsilon-\frac{2 q-(p-1) N}{N(p-1)} r^{q}\right]=\frac{2(p+1)}{N(p-1)} \int_{0}^{\infty} r^{N-1} u^{\prime 2}
$$

This proves (15) in the case $N=1,2$. To obtain a sharper inequality for dimensions $N \geq 3$, we derive another identity as follows. Differentiating (8) with respect to $r$ we obtain

$$
\frac{1}{r^{N-1}}\left(r^{N-1} u^{\prime \prime}\right)^{\prime}-\frac{N-1}{r^{2}} u^{\prime}-u^{\prime}+\left(\varepsilon+r^{q}\right) p u^{p-1} u^{\prime}+q r^{q-1} u^{p}=0 .
$$


Multiplying (18) by $r^{N-1} u$ integrating on $[0, \infty]$, and using integration by parts we get

$$
\int_{0}^{\infty}\left(u^{\prime} r^{N-1}\right)^{\prime} u^{\prime}+(N-1) r^{N-3} u u^{\prime}-r^{N-1} u u^{\prime}+\left(r^{q}+\varepsilon\right) r^{N-1} p u^{p} u^{\prime}+q r^{q-1} u^{p+1} r^{N-1}=0 .
$$

Using (8) and rearranging we obtain

$$
\int_{0}^{\infty} r^{N-1}(p-1)\left(r^{q}+\varepsilon\right) u^{p} u^{\prime}+q \int_{0}^{\infty} r^{N-2+q} u^{p+1}=-\frac{(N-1)}{2} \int_{0}^{\infty} r^{N-3}\left(u^{2}\right)^{\prime}
$$

Note that

$$
\int_{0}^{\infty} r^{N-1+q} u^{p} u^{\prime}=-\frac{N-1+q}{p+1} \int_{0}^{\infty} r^{N-2+q} u^{p+1}
$$

and moreover,

$$
-\int_{0}^{\infty} r^{N-3}\left(u^{2}\right)^{\prime}=\left\{\begin{array}{c}
(N-3) \int_{0}^{\infty} r^{N-4} u^{2}, \quad N>4 \\
u(0)^{2}, \quad N=3
\end{array} \quad>0 .\right.
$$

Thus we obtain

$$
\int_{0}^{\infty} r^{N-1} u^{p+1}\left[\varepsilon-\left(\frac{2 q-(p-1)(N-1)}{p+1}\right) r^{q}\right]=-\int_{0}^{\infty} r^{N-3}\left(u^{2}\right)^{\prime}>0, \quad N \geq 3 .
$$

This proves (15) for dimension $N \geq 3$.

Step 2. Given $q$ that satisfies (9), note that (15) holds with $c_{1}>0$. We now show that there exists a constant $C$ such that $u(0)>C \varepsilon^{-1 /(p-1)}$ for all sufficiently small $\varepsilon$. Let $r_{0}=\left(1 / c_{1}\right)^{1 / q} \varepsilon^{1 / q}$ be the root of $\varepsilon-c_{1} r^{q}=0$. Then

$$
\int_{0}^{\infty} r^{N-1} u^{p+1}\left[\varepsilon-c_{1} r^{q}\right]=\int_{0}^{r_{0}} r^{N-1} u^{p+1}\left[\varepsilon-c_{1} r^{q}\right]-\int_{r_{0}}^{\infty} r^{N-1} u^{p+1}\left[c_{1} r^{q}-\varepsilon\right]>0
$$

so that

$$
\int_{0}^{r_{0}} r^{N-1} u^{p+1}\left[\varepsilon-c_{1} r^{q}\right]>\int_{r_{0}}^{\infty} r^{N-1} u^{p+1}\left[c_{1} r^{q}-\varepsilon\right]>\int_{r_{1}}^{r_{1}+r_{0}} r^{N-1} u^{p+1}\left[c_{1} r^{q}-\varepsilon\right] .
$$

for any $r_{1} \geq r_{0}$. In particular, choose $r_{1}$ to satisfy $\varepsilon-c_{1} r^{q}=-\varepsilon$, i.e. $r_{1}=\left(2 / c_{1}\right)^{1 / q} \varepsilon^{1 / q}$. Then $\varepsilon \geq \varepsilon-c_{1} r^{q}$ on $\left[0, r_{0}\right]$ and $c_{1} r^{q}-\varepsilon \geq \varepsilon$ on $\left[r_{1}, r_{1}+r_{0}\right]$ so that

$$
\int_{0}^{r_{0}} r^{N-1} u^{p+1}>\int_{r_{1}}^{r_{1}+r_{0}} r^{N-1} u^{p+1} .
$$

It follows that $r^{N-1} u^{p+1}$ cannot be increasing on $\left[0, r_{0}+r_{1}\right]$. In particular, $u$ cannot be increasing on $\left[0, C_{1} \varepsilon^{1 / q}\right]$ where $C_{1}=\left(2 / c_{1}\right)^{1 / q}+\left(1 / c_{1}\right)^{1 / q}$. Now consider the initial value problem

$$
0=\hat{u}_{r r}+\frac{N-1}{r} \hat{u}_{r}-\hat{u}+\hat{u}^{p}\left(\varepsilon+r^{q}\right) ; \quad \hat{u}(0)=\xi, \quad \hat{u}^{\prime}(0)=0 .
$$

We claim that there exists a constant $C_{2}$ such that $\hat{u}$ is non-decreasing on the interval $\left[0, C_{1} \varepsilon^{1 / q}\right]$ whenever $\xi<C_{2} \varepsilon^{-1 /(p-1)}$. In fact, note that by comparison principle, $\hat{u}<\xi v$ where $v$ solves $v_{r r}+\frac{N-1}{r} v_{r}-v=0, v^{\prime}(0)=0, v(0)=1$. It follows that $\hat{u}<\xi C_{0}$ on $[0,1]$ where $C_{0}=v(1)$ is some 
constant independent of $\varepsilon, p, q, \xi$. Now suppose that $u$ is increasing on $\left[0, r_{m}\right]$ and has a maximum at $r_{m}<C_{1} \varepsilon^{1 / q}$. At such a point,

$$
\varepsilon+r_{m}^{q}=\frac{1}{\hat{u}^{p-1}}-\frac{u^{\prime \prime}\left(r_{m}\right)}{\hat{u}^{p}} \geq \frac{C_{0}^{1-p}}{\xi^{p-1}}
$$

where $C_{1}=C_{0}^{1-p}$. It follows that

$$
r_{m} \geq\left(\frac{C_{0}^{1-p}}{\xi^{p-1}}-\varepsilon\right)^{1 / q}>C_{1} \varepsilon^{1 / q}
$$

whenever

$$
\xi<\frac{C_{0}^{-1}}{\left(C_{1}^{q}+1\right)^{\frac{1}{p-1}} \varepsilon^{1 /(p-1)}} .
$$

Therefore $\hat{u}$ is increasing on $\left[0, C_{1} \varepsilon^{1 / q}\right]$ whenever $\xi<C_{2} \varepsilon^{-1 /(p-1)}$ where $C_{2}=\frac{C_{0}^{-1}}{\left(C_{1}^{q}+1\right)^{\frac{1}{p-1}}}$. It follows that $u(0)>C_{2} \varepsilon^{-1 /(p-1)}$.

Step 3. We claim that there exists a number $\xi_{0}$ such that for all $\varepsilon<1$ and all $\xi>\xi_{0}$, the solution $\hat{u}$ to $(22)$ crosses the $x$-axis. To see this, let

$$
\hat{u}=\xi v ; \quad r=\xi^{\frac{1-p}{q+2}} s .
$$

Then (22) becomes

$$
v_{s s}+\frac{(N-1)}{s} v_{s}+s^{q} v^{p}=\delta\left(v-\varepsilon v^{p}\right) ; \quad v(0)=1, v^{\prime}(0)=0
$$

where

$$
\delta=\xi^{-\left(\frac{p-1}{q+2} q\right)} .
$$

Now by Lemma 8, the solution to (23) with $\delta=0$ crosses zero, provided that $q$ satisfies (9). By continuity, it follows that $v$ also crosses zero for all $\delta<\delta_{0}$, for some $\delta_{0}>0$; hence $\xi>\delta_{0}^{-\left(\frac{q+2}{q(p-1)}\right)}$. This proves the claim.

Step 4. Let $\varepsilon_{0}=\min \left\{1,\left(\frac{C_{2}}{\xi_{0}}\right)^{p-1}\right\}$. Suppose that there exists solution to (8) with $\varepsilon<\varepsilon_{0}$. Then from Step 2, we have that $u(0)>\xi_{0}$. But then by Step 3, $u(x)$ will cross the $x$-axis, a contradiction to the assumption that $u>0$ for all $x$. This concludes the proof of statement (i). To prove (ii), note that in the case $\varepsilon=0, q=q_{c}$, the identity (21) reduces to $0=-\int_{0}^{\infty} r^{N-3}\left(u^{2}\right)^{\prime}$, which contradicts (20).

Step 5. We now discuss the existence results with $\varepsilon=0$ and $N \geq 3$. If $p \in\left(1, p^{\star}\right)$ where $p^{\star}=\frac{N+2}{N-2}$ is the critical exponent, then the existence is an immediate consequence of a more general result proven in [1], whose statement we reproduce here for reader's convenience. Namely, consider the more general problem

$$
0=u_{r r}+\frac{N-1}{r} u_{r}-u+u^{p} h(r)
$$


Then Corrollary 4.8 of [1] implies that solution to (24) exists provided that $p \in\left(1, p^{\star}\right)$ and $|h(r)|<C+r^{q}$ for some constant $C>0,0<q<q_{c}$, for all $r \geq 0$. In fact, their proof carries through in the more general case where $p>1, q_{\star}<q<q_{c}$ and $q \geq 0$ (note that $q_{\star}<0$ iff $p<p^{\star}$ ). For completeness, this proof is included in Appendix B. We remark that the necessary condition $q<q_{c}$ follows immediately from (15) with $\varepsilon=0$; the condition $q_{\star}<q$ is the result of combining Pohozhaev identities (16), (17) with $\varepsilon=0$,

$$
\int r^{N-1} u^{2}+\left(-1+\frac{N}{2}-\frac{N+q}{p+1}\right) \int_{0}^{\infty} r^{N-1+q} u^{p+1}=0
$$

so that $-1+\frac{N}{2}-\frac{N+q}{p+1}>0 \Longleftrightarrow q_{\star}<q$.

Next we show uniqueness when $q \in\left(q_{\star}, q_{c}\right)$ and $\varepsilon=0$. We follow the method outlined in [17], which works for more general equations of the form (24). Make a change of variables

$$
u(r)=v(s) g(r)
$$

where $s=s(r)$ is to be specified shortly. We have

$$
\begin{aligned}
u_{r} & =v_{s} \frac{d s}{d r} g+v g^{\prime} \\
u_{r r} & =v_{s s}\left(\frac{d s}{d r}\right)^{2} g+2 v_{s} g^{\prime} \frac{d s}{d r}+v_{s} \frac{d^{2} s}{d r^{2}} g+v g^{\prime \prime}
\end{aligned}
$$

so that (24) becomes

$$
v_{s s}\left(\frac{d s}{d r}\right)^{2} g+v_{s}\left(2 g^{\prime} \frac{d s}{d r}+\frac{d^{2} s}{d r^{2}} g+\frac{N-1}{r} \frac{d s}{d r} g\right)+v\left(g^{\prime \prime}+\frac{N-1}{r} g^{\prime}-g\right)+v^{p} g^{p} h=0
$$

Next choose $s$ so that

$$
\frac{d^{2} s}{d r^{2}}=-\frac{d s}{d r}\left(2 \frac{g^{\prime}}{g}+\frac{N-1}{r}\right)
$$

so that

Also choose $g$ so that

$$
\frac{d s}{d r}=g^{-2} r^{-(N-1)}
$$

$$
\begin{aligned}
g^{p} h & =\left(\frac{d s}{d r}\right)^{2} g=g^{-3} r^{-2(N-1)} \\
g & =h^{\frac{1}{-3-p}} r^{\frac{2(N-1)}{-3-p}}
\end{aligned}
$$

We then get

$$
v_{s s}+F(r) v+v^{p}=0
$$

where

$$
F(r)=\left(g^{\prime \prime}+\frac{N-1}{r} g^{\prime}-g\right) g^{3} r^{2(N-1)} ; \quad g=h^{\frac{1}{-3-p}} r^{\frac{2(N-1)}{-3-p}}
$$


For the equation (25), Theorem 1 of [17] guarantees uniqueness, provided that $F(r)$ satisfies the so called $\Lambda$-property on $(0, \infty)$; that is $F(r)$ has at most one maximum and no interior minimum. It remains to verify this property.

Note that

$$
q_{c}-q_{\star}=\frac{p+3}{2}
$$

This suggests a change of variables,

$$
\delta:=\left(q_{c}-q\right) \frac{2}{p+3} .
$$

Then

$$
q \in\left(q_{\star}, q_{c}\right) \Longleftrightarrow \delta \in(0,1)
$$

and using $h=r^{q}, F(r)$ becomes

$$
F(r)=-c_{1} r^{2(-1+\delta)}-r^{2 \delta}, \text { where } c_{1}:=(N-1-\delta)(N-3+\delta) / 4>0 .
$$

Provided that (27) holds, it is clear that $F^{\prime \prime}(r)<0$, so that $F(r)$ indeed has the $\Lambda$-property. Therefore Theorem 1 of [17] proves the uniqueness of solution to (8) with $\varepsilon=0$ provided $q \in\left(q_{\star}, q_{c}\right)$.

Theorem 1 provides conditions for when the bifurcation curve is bounded and thus shows an existence of the fold point under the conditions (3). To obtain a more refined information, we examine what happens to the bifurcation curve when $u(0)$ is small. In this case, there may exist solutions to (4) which attain maximum far away from the origin. These are studied using formal asymptotics in the Appendix A. In dimensions $N \geq 2$, this analysis also leads to the threshold $q=q_{c}$.

\section{Stability analysis}

We now study the stability of the time-dependent problem (1). It is convenient to consider a more general problem,

$$
\left\{\begin{array}{l}
u_{t}=\Delta u-u+u^{p} h(x ; a) \frac{c_{0}}{\int u^{p+1} h(x ; a)} ; \quad x \in \mathbb{R}^{N} \\
\nabla u(0, t)=0 ; \quad u \rightarrow 0 \text { as }|x| \rightarrow \infty
\end{array}\right.
$$

where $h(x)=h(r ; a)$ is radially symmetric function depending on the parameter, $a$; the model (1) corresponds to $h=1+a r^{q}$. The constant $c_{0}$ is chosen so that the time-independent solution is the ground state satisfiying

$$
u_{0 r r}+\frac{N-1}{r} u_{0 r}-u_{0}+u_{0}^{p} h(r ; a)=0, \quad u_{0}^{\prime}(0)=0, \quad u_{0} \rightarrow 0 \text { as } r \rightarrow \infty, \quad u_{0}>0 ;
$$

that is,

$$
c_{0}=\int u_{0}^{p+1} h .
$$

Since the constant $c_{0}$ can be scaled out by scaling $u$, its inclusion does not change the stability properties. 
The condition $\nabla u(0, t)=0$ will be necessary to avoid translational instabilities. Equivalently, we may simply restrict (28) to the positive quadrant $\Omega=\left\{\left(x_{1}, x_{2}, \cdots x_{N}\right): x_{i}>0, i=1 \ldots N\right\}$ and impose Neumann boundary conditions on $\partial \Omega$. In this setting, the spike solution at the center becomes a boundary spike at the corner of $\Omega$.

When $h=1$, the problem (28) and its generalizations are sometimes referred to as the shadow system [28]. It naturally occurs in the high diffusivity ratio limit of some reaction-diffusion systems, for example Gierer-Meinhardt model [27], and Gray-Scott model [20], [3]. The main feature of (28) with $h=1$ is that the integral term in the denominator stabilizes the large eigenvalues [28].

We begin our investigation by linearizing around the steady state. Set

$$
u(x, t)=u(r)+e^{\lambda t} Z(x) .
$$

where $u(r)$ satisfies $(29)$ (here and below we drop the subscript 0 for convenience) and $Z \ll 1$. Define

$$
L Z:=\Delta Z-Z+u^{p-1} h p Z .
$$

Then we have

$$
\left\{\begin{array}{c}
\lambda Z=L Z-u^{p} h \frac{(p+1)}{c_{0}} \int Z u^{p} h \\
\nabla Z(0)=0 ; \quad Z \rightarrow 0 \text { as }|x| \rightarrow \infty
\end{array}\right.
$$

In one dimension the condition $Z^{\prime}(0)=0$ assures that $Z$ is even (i.e. radially symmetric) eigenfunction. In dimensions $N \geq 2$, the problem (31) has radially symmetric eigenfunction; but may also have non-radially symmetric modes. We start by studying radially symmetric perturbations.

3.1. Radially symmetric perturbations. In this section we examine the radial stability of (28). That is, we consider solutions $(Z, \lambda)$ to $(31)$, where $Z$ is restricted to the space of radially symmetric functions. As before, let

$$
s=u(0 ; a)
$$

where $u(x ; a)$ is the ground state solution to $(29)$. We also assume that

$$
h(x ; 0)=1 ; \quad p \in\left(1, p^{\star}\right) \text { if } N \geq 3 \text { or } p>1 \text { if } N=1 \text { or } 2 .
$$

Then there is a unique value $s_{0}$ with $a=0$ which corresponds to the unique ground state solution to (29) with $h=1$ [17]. Now consider the bifurcation curve $(s, a(s))$ going through $s=s_{0}, a=0$. Suppose that such curve has a fold point (Conditions (3) are sufficient when $h=1+a r^{q}$; see some examples in Figure 2). Our main result here to show Condition (S3) in one dimension. In addition, we will show that the even eigenfunction at the fold point of (31) corresponding to a zero eigenvalue has a root; this will prove Condition (S2). In order to show this, we need to assume the following.

Condition 3 (Non-degeneracy Condition). Let $u_{s}=\partial u / \partial s$ where $s$ is given by (32) The following conditions are equivalent.

(i) The equation $L Z=0$ admits a nonzero radially symmetric solution.

(ii) $\frac{\partial a}{\partial s}=0$.

(iii) $L u_{s}=0$. 


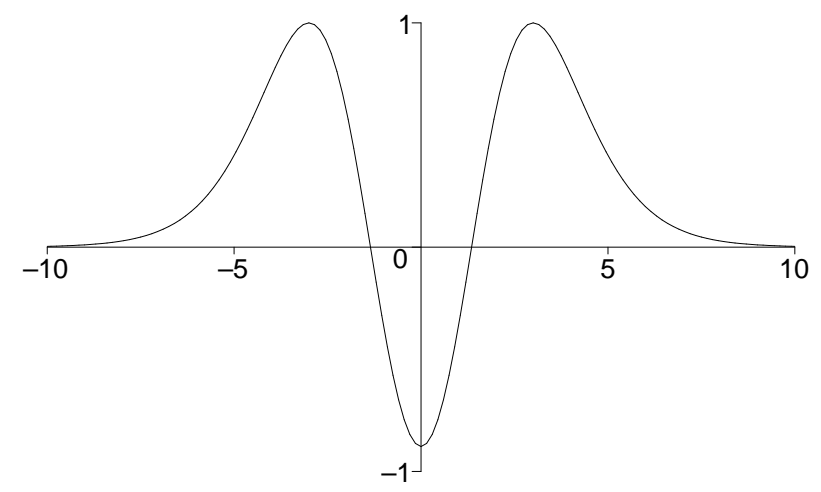

(a)

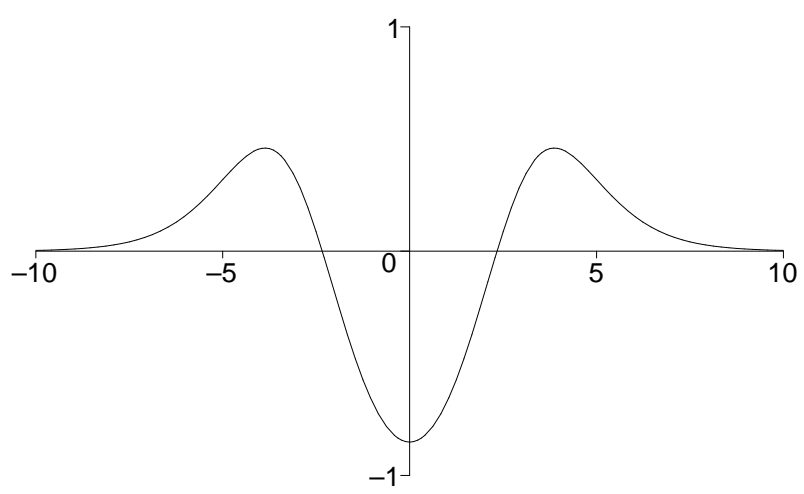

(b)

FiguRE 3. (a) The "dimple" eigenfunction at the fold point, corresponding to the zero eigenvalue of (1) with $N=1, p=2, q=2, a=0.079$. The shape of the eigenfunction is responsible for pulse replication. (b) The "dimple" eigenfunction for the reduced Gray-Scott model (2), $N=1$, taken from [14].

Note that $u_{s}$ satisfies

$$
L u_{s}=-u^{p} h_{a} \frac{\partial a}{\partial s} .
$$

It immediately follows that $(i i) \Longrightarrow(i i i) \Longrightarrow(i)$. It is unclear under what assumptions on $h$ one can show that $(i) \Longrightarrow(i i)$; generally, we have resorted to numerics to verify this numerically for specific choices of $h(r)$. We were unable to find any example of $h(x ; a)$ which would contradict the Condition 3.

We now state the main result.

Theorem 4. Suppose that $h(r ; 0)=1$ and let $s=u(0 ; a)$ where $u(x ; a)$ is the ground state solution to (29). Supopse the bifurcation curve $a=a(s)$ has the following properties:

(i) $a\left(s_{0}\right)=0$ for some $s_{0}$;

(ii) $a^{\prime}\left(s_{c}\right)=0$ for some $s_{c}$ and $a^{\prime}(s) \neq 0$ for all $s \in\left(s_{c}, s_{0}\right]$.

If $s=s_{c}$ then (31) admits a zero eigenvalue whose eigenfunction is given by $Z=\left.\frac{\partial u}{\partial s}\right|_{s=s_{c}}$. Moreover $Z(r)$ has at least one root $r>0$. Thus Condition (S2) is proven.

Let $s \in\left(s_{c}, s_{0}\right]$, and suppose Condition 3 is satisfied. Then the corresponding nonlocal eigenvalue problem (31) is stable with respect to radially symmetric perturbations.

An example of the eigenfunction $\left.\frac{\partial u}{\partial s}\right|_{s=s_{c}}$ with $N=1, p=2, q=2$ is shown in Figure 3 . The pulse-splitting as observed in Figure 1(a) is due to its "upside-down Mexican-hat" shape.

Note that Theorem 4 provides a partial generalization of [28], where the case $h=1$ was proven ${ }^{1}$. Theorem 4 relies on the following lemma.

\footnotetext{
${ }^{1}$ In [28], the stabilify of the problem

$$
u_{t}=\Delta u-u+u^{p} \frac{1}{\int u^{m}}
$$

was considered; the case $h=1$ in (28) corresponds to $m=p+1$ ).
} 
Lemma 5. Suppose that the non-degeneracy condition 3 is satisfied. Consider the local radially symmetric eigenvalue problem

$$
L \Phi=\lambda \Phi ; \quad \Phi \text { is radially symmetric }
$$

and the corresponding nonlocal problem,

$$
\lambda Z=L Z-u^{p} h \frac{(p+1)}{c_{0}} \int Z u^{p} h ; \quad Z \text { is radially symmetric }
$$

Suppose (35) admits a unique positive eigenvalue. Then the nonlocal problem (36) is stable, i.e. it has no positive eignevalues. Suppose (35) admits at least two positive eigenvalues. Then the nonlocal eigenvalue problem (31) is unstable, i.e. it admits at least one positive eigenvalue.

Proof. We will rely on the following identity:

$$
L u=u^{p} h(p-1) .
$$

Note that the eigenvalue problem (36) is self-adjoint so that the eigenvalues are all purely real. There are two cases to consider. First, suppose that

$$
\int Z u^{p} h \neq 0
$$

Then we may scale $Z$ so that (36) becomes

$$
(L-\lambda) Z=u^{p} h ; \quad \int Z u^{p} h=\frac{c_{0}}{p+1} .
$$

Define

$$
f(\lambda):=\int(L-\lambda)^{-1}\left[u^{p} h\right] u^{p} h .
$$

Then (39) becomes

$$
f(\lambda)=\frac{c_{0}}{p+1}
$$

We compute

$$
\begin{aligned}
f^{\prime}(\lambda) & =\int(L-\lambda)^{-2}\left[u^{p} h\right] u^{p} h \\
& =\int\left\{(L-\lambda)^{-1}\left[u^{p} h\right]\right\}^{2}
\end{aligned}
$$

so that $f$ is always increasing. Also note that $f(\lambda)$ has a singularity at every positive eigenvalue of the local problem (35). Suppose that (35) admits $K$ positive eigenvalues, $K \geq 1$. Then $f(\lambda)$ has $K$ vertical asymptotes for positive $\lambda$. Now from (37) we note that

$$
f(0)=\int \frac{u}{p-1} u^{p} h=\frac{c_{0}}{p-1}
$$

so that $f(0)>\frac{c_{0}}{p+1}$. Moreover, $f(\lambda) \rightarrow 0$ as $\lambda \rightarrow 0$. Thus there are precisely $K-1$ positive solutions to $(40)$. 
We have shown that if $K \geq 2$ then (36) is unstable. It remains to show that (36) is stable when $K=1$. Then there are no positive eigenvalues of (36) that satisfy (38). It remains to consider the case $\int Z u^{p} h=0 ; \quad K=1$. But then $Z$ satisfies $L Z=\lambda Z$. Thus $\lambda=\lambda_{1}$, where $\lambda_{1}$ is the unique positive eigenvalue of (35). Now multiplying (37) by $Z$ and integrating, we then obtain $\lambda_{1} \int u Z=0$. Since we assumed $\lambda_{k} \neq 0$, and $u>0$, this means that $Z$ must change sign. But this contradicts the fact that $Z$ is is the eigenfunction of the principal eigenvalue of the local problem (35).

Proof of 4. First, note that when $a=0, s=s_{0}$, we have $h(x)=1$. In this case, the problem $L Z=0$ admits $N$ independent solutions given by $Z_{k}=\hat{e}_{k} u^{\prime}(r), k=1 \ldots N$ where $\hat{e}_{k}$ is the $k$-th unit vector and $u(r)$ is the radially symmetric ground state solution to (29) with $h=1$. Thus the local eigenvalue problem $L Z=\lambda Z$ admits $N$ eigenfunctions corresponding to a zero eigenvalue. Moreover it is well known that $u(r)$ is unique and is a decreasing function [17]. It follows that the nodal set $\left\{x: Z_{k}=0\right\}$ is precisely $\left\{x: x_{k}=0\right\}$, which divides $\mathbb{R}^{N}$ into exactly two connected sets. By oscillation theorem there must be a positive eigenvalue whose eigenfunction has no root; such an eigenvalue is unique and the corresponding eigenfunction is radially symmetric; all other radially symmetric eigenfunctions correspond to strictly negative eigenvalues. This proves that (35) admits precisely one positive eigenvalue when $s=s_{0}$. Next, note that the eigenvalues are all real since 36 is self-adjoint. By Condition 3, the eigenvalues cannot be zero for $s \in\left(s_{c}, s_{0}\right)$. By continuity it follows that (35) admits exactly one positive eigenvalue for all $s \in\left(s_{c}, s_{0}\right]$. By Lemma 5 , it then follows that (36) is stable.

We now prove that $u_{s}=\partial u / \partial s$ is an eigenfunction of (36) corresponding to $\lambda=0$ whenever $s=s_{c}$. Certainly $L u_{s}=0$ (see Condition 3 ). We now show that

$$
\int u_{s} u^{p} h=0
$$

so that $u_{s}$ is indeed an eigenfunction of (36) corresponding to $\lambda=0$. This follows by multiplying the identity (37) by $u_{s}$ and then integrating by parts and using $L u_{s}=0$. Equation (41) also shows that $u_{s}$ has a strictly positive root since $h, u>0$.

3.2. Nonradial perturbations in three dimensions. Theorem 4 shows that the top branch of the bifurcation curve is stable with respect to radially symmetric perturbations. This implies full stability in one dimension. However in higher dimensions, non-radial instabilities can and do occur. In this study such instabilities in three dimensions. As before, the starting point is the eigenvalue problem (31). We then use spherical coordinates

$$
\begin{gathered}
x=r \sin \theta \cos \phi, \quad y=r \sin \theta \sin \phi, \quad z=r \cos \theta \\
\Delta Z=Z_{r r}+\frac{2}{r} Z_{r}+\frac{1}{r^{2}}\left(\frac{1}{\sin ^{2} \theta} Z_{\phi \phi}+\frac{1}{\sin \theta}\left(\sin \theta Z_{\theta}\right)_{\theta}\right)
\end{gathered}
$$

We decompose the eigenfunction as

$$
Z(x, y, z)=\Phi(r) Y_{l}^{m}(\theta, \phi) ; l=0,1, \ldots ; \quad m=0, \pm 1 \ldots \pm l
$$

where $Y_{l}^{m}$ are the spherical harmonics (See for example Chapter 10 of [26]). Now note that $Y_{0}^{0}=1$ so that by orthogonality property of spherical harmonics, we have $\int Y_{l}^{m}=0, l \geq 1$ and $\int h Z u^{p-1}=0$. 
In particular the nonlocal term in (31) disappears so that $\Phi$ satisfies

$$
\lambda_{l} \Phi=\Phi_{r r}+\frac{2}{r} \Phi_{r}-\frac{\gamma}{r^{2}} \Phi+p h u^{p-1} \Phi ; \quad \gamma=l(l+1), l \geq 1 .
$$

Note that the case $l=0$ corresponds to the radially symmetric eignefunctions whose stability was already characterized by Theorem 4 . The case $l=1$ corresponds to translational modes; in such a case $Y_{1}^{m}=x / r, y / r$ or $z / r$. In particular, if $l=1, h=1$ then the solution is $\lambda_{1}=0, \Phi=u_{r}$. In general, $\lambda_{1}$ is typically unstable. It is for this reason that we have imposed the condition $\nabla u(0, t)=0$, in $(28)$; so the translational modes $l=1$ are inadmissable (they do not satisfy $\nabla Z(0)=0)$. So we need to only consider the stability of non-radial nodes $l \geq 2$. To geth some insight, let us consider the case $h=1+a r^{q}$ with $q \geq q_{c}$. where $q_{c}$ is given in (7b). In Appendix A.2 (for $q>q_{c}$ ) and Appendix A.3 (for $q=q_{c}$ ) we have constructed a ring-like solution with $s=u(0) \rightarrow 0$, either for $q=q_{c}$ or $q>q_{c}$. Such solutions have the form

$$
u(r) \sim C w(y) \text { where } y=r-r_{0}, \quad r_{0} \gg 1
$$

where $C=\left(a r_{0}^{q}\right)^{1 /(1-p)}$ and $w(y)$ is the one-dimensional ground state that satisfies (44). Since $w$ decays exponentially away from $r_{0}$, to leading order we have $\frac{2}{r} \phi_{r}-\frac{\gamma}{r^{2}} \phi \sim O\left(\frac{1}{r_{0}}\right)$ so that

$$
\lambda_{l} \phi \sim \phi_{y y}-\phi+p w^{p-1} \phi .
$$

It is well-known that (42) admits a positive eigenvalue (in fact, it is a special case of 35 with $N=1$ and $h=1)$. This proves that $\lambda_{l}>0$ for $l \geq 2$ if $u(0)$ is sufficiently small. In particular, as the bifurcation curve is traversed in the direction of decreasing $s$, the mode $l=2$ eventually becomes unstable. This is illustrated in Figure 2(b).

Due to ordering principle for the local eigenvalue problem $L Z=\lambda Z$, the eigenvalues are ordered $\lambda_{2} \geq \lambda_{3} \geq \lambda_{4} \geq \cdots$. However no such ordering exists between the radial eigenvalue $\lambda_{r}$ and $\lambda_{2}$, since $\lambda_{r}$ satisfies the non-local problem (31). This leads to the following question: As the bifurcation curve is traversed starting with $a=0, u(0)=O(1)$, can the nonradial mode $\lambda_{2}$ become unstable before the radial mode $\lambda_{r}$ ? Since $\lambda_{r}$ becomes unstable at the fold point, the answer is yes provided that the bifurcation curve has no fold point. In particular, if the solution to (4) is unique for all $a>0$, then the fold point does not exist. We now show that this is the case when $p=2$ and $q=q_{c}=1$. Using Theorem 1 of [17], the solution is unique if the function $F(r)$ given by (26) with $h(r)=1+$ ar satisfies the $\Lambda$ property (as described below the equation (26)). After some algebra we simplify to obtain

$$
\begin{aligned}
& F(r)=-r^{-6 / 5}(1+a r)^{-14 / 5}\left(r^{4} a^{2}+2 r^{3} a+r^{2}+\frac{2}{5} a r+\frac{4}{25}\right) \\
& F^{\prime}(r)=\frac{-2}{125} r^{-6 / 5}(1+a r)^{-14 / 5}\left(25 r^{4} a^{2}+50 r^{3} a-\left(75 a^{2}-50\right) r^{2}-45 a r-12\right)
\end{aligned}
$$

Now clearly, $F \rightarrow-\infty$ as $r \rightarrow 0^{+}$. So to show the $\Lambda$ property, it suffices to show that $F^{\prime}=0$ has a unique solution. But this follows from Descartes rule of signs, since the coefficients in the polynomial inside $F^{\prime}(r)$ change sign precisely once.

To summarize, in the case $p=2, q=q_{c}=1$, the radial mode $\lambda_{r}$ is stable for all $a>0$; however the nonradial mode $\lambda_{2}$ becomes unstable for sufficiently large $a$. 
When $p=2, q>1$, the bifurcation curve has a fold point, where $\lambda_{r}=0$. In general it is unknown whether $\lambda_{2}$ becomes unstable before $\lambda_{r}$ or vice-versa, as $a$ is increased. However if $p=2$ and $q$ is close to 1 then because of continuous dependence on parameters, $\lambda_{2}$ is destabilized before $\lambda_{r}$ as $a$ is increased. Numerically, we observe that the opposite is true if $q$ is sufficiently large as the following two tables illustrate.

\begin{tabular}{|l|l|l|l|}
\hline \multicolumn{4}{|c|}{$p=2, q=1.3$} \\
\hline$a$ & $s$ & $\lambda_{r}$ & $\lambda_{2}$ \\
\hline 0.0000 & 4.1895 & -0.79 & -1.03 \\
\hline 0.1104 & 3.1895 & -0.62 & -1.02 \\
\hline 0.2311 & 2.2895 & -0.44 & -0.67 \\
\hline 0.4410 & 1.1395 & -0.18 & -0.02 \\
\hline 0.4523 & 1.0895 & -0.17 & 0.00 \\
\hline 0.6044 & 0.3895 & -0.005 & 0.59 \\
\hline $\mathbf{0 . 6 0 4 6}$ & $\mathbf{0 . 3 3 9 5}$ & $\mathbf{0 . 0 0 5}$ & 0.65 \\
\hline 0.5981 & 0.2895 & 0.014 & 0.71 \\
\hline 0.4370 & 0.0895 & 0.026 & 0.98 \\
\hline 0.1647 & 0.001 & 0.0067 & 1.19 \\
\hline
\end{tabular}

\begin{tabular}{|l|l|l|l|}
\hline \multicolumn{4}{|c|}{$p=2, q=3$} \\
\hline$a$ & $s$ & $\lambda_{r}$ & $\lambda_{2}$ \\
\hline 0.0000 & 4.1895 & -0.79 & -1.037 \\
\hline 0.0183 & 3.6395 & -0.54 & -0.99 \\
\hline 0.0343 & 2.5895 & -0.024 & -0.3 \\
\hline $\mathbf{0 . 0 3 4 4}$ & $\mathbf{2 . 5 3 9 5}$ & $\mathbf{0 . 0 0 1 5}$ & -0.27 \\
\hline 0.0343 & 2.4895 & 0.027 & -0.23 \\
\hline 0.0326 & 2.1895 & 0.18 & 0.00 \\
\hline 0.0314 & 2.0895 & 0.23 & 0.066 \\
\hline 0.0229 & 1.6395 & 0.42 & 0.39 \\
\hline 0.0128 & 1.1395 & 0.46 & 0.66 \\
\hline 0.0003 & 0.001 & 0.033 & 1.19 \\
\hline
\end{tabular}

For $p=2$ and a given $q$, these two tables list the values of $\lambda_{r}$ and $\lambda_{2}$ as well as $a=a(s)$, computed numerically. Starting with $a=0 \Longrightarrow s=4.1895$, we followed the bifurcation curve in the direction of decreasing $s$. When $q=1.3$, the fold point occurs at $a \sim 0.6046$; numerics confirm that the radial node $\lambda_{r}$ crosses zero at that point (see also Theorem 4). However the non-radial mode $\lambda_{2}$ becomes unstable at around $a \sim 0.4523$ on the top branch of the bifurcation curve. Hence in this case, the mode $\lambda_{2}$ becomes unstable before $\lambda_{r}$ as $a$ is increased from $a=0$. When $q=3$, the opposite behaviour is observed: the fold point occurs at $a \sim 0.0344$ whereas the non-radial mode $\lambda_{2}$ is destabilized only on the bottom branch of the bifurcation curve. In particular the top branch of the bifurcation curve is stable with respect to $\lambda_{2}$ (and hence, stable with respect to all non-radial perturbations due to the ordering property). This is also illustrated in Figure 2(b), where the bifurcation curve is plotted along the threshold values of $a$ when $\lambda_{r}=0$ or when $\lambda_{2}=0$, for several different values of $q$ with $p=2$.

\section{Discussion}

In this paper, we have shown that even a single PDE with heterogenuity has the same selfreplication behaviour as more complicated reaction-diffusion systems, such as Gray-Scott. For our simpler model, we are able to prove analytically Nishiura-Uyema Conditions (S1) and (S2). For condition (S3), we required an additional non-degeneracy condition (3). While we were unable to prove this condition, it is easy to verify it numerically; furthermore it reduces the nonlocal eigenvalue problem (31) to a computation which does not involve nonlocal term. Theoretically, it is an open question as to what assumptions on $h(r ; a)$ are necessary to prove the non-degeneracy condition 3

In Gray-Scott model, peanut-splitting is the dominant self-replication mechanism in two dimensions as observed by [23], [19], [20]. On the other hand, it was observed numerically in [15] that 
either the radial or peanut-type instability can be dominant in the Gierer-Meinhardt model in two dimensions, depending on pararameter values. Our simplifed model has a similar structure: either instability is possible, depending on how the parameters $p, q$ are chosen.

In this paper we prove the first rigorous result about replication in three dimensions. As of now, there are no analytical results about replication in three dimensions (but see [19], [20] for some numerical results on (2) in three dimensions).

We conclude with the following conjecture, which is a generalization of Corrollary 4.8 in [1].

Conjecture 6. Consider the system

$$
0=\Delta u-u+u^{p} h(r) ; \quad u>0, u \rightarrow 0 \text { as } r \rightarrow \infty .
$$

Suppose $p>1$ and $h(r)$ satisfies

$$
|h(r)| \leq C\left(1+r^{q}\right) \quad \text { where } q \geq 0 \text { and } q \in\left(q_{\star}, q_{c}\right)
$$

where $C$ is some constant and $q_{\star}, q_{c}$ are given by (7). Then (43) has a radially symmetric solution.

In [1], Corrollary 4.8, this result was shown under a more restrictive assumption $p \in\left(1, p^{\star}\right)$, in which case $q_{\star}<0$. Here, we dont assume that $p<p^{\star}$; this assumption is replaced with the more general assumption $q>q_{\star}$.

\section{Appendix A. Asymptotic Analysis of (4) With Small $u(0)$}

We now examine the behaviour of the solution with small $u(0)$. The analysis is different for $N=1$ or $N \geq 2$.

One dimension. We consider (4) with $N=1$, in the limit $a \ll 1$ :

$$
u_{x x}-u+u^{p}\left(1+a x^{q}\right)=0 ; \quad a \ll 1 ; \quad u^{\prime}(0)=0 ; \quad u>0 ; \quad u \rightarrow 0 \text { as } x \rightarrow \infty .
$$

We seek solutions of the form

$$
u(x) \sim w(y)+R(x) ; \quad y=x-x_{0} ; \quad x_{0} \gg 0, \quad R \ll 1 .
$$

where $w(y)$ is the (unique) one-dimensional ground state of the homogeneous problem,

$$
w_{y y}-w+w^{p}=0 ; \quad w^{\prime}(0)=0, \quad w>0, \quad w \rightarrow 0 \text { as }|y| \rightarrow \infty .
$$

and $R$ is the small remainder term. Then $R$ satisfies

$$
R_{y y}-R+p w^{p-1} R+a x^{q} U^{p}=0 .
$$

Note also that

$$
\left(w_{y}\right)_{y y}-w_{y}+p w^{p-1} w_{y}=0 .
$$

Multiplying (45) by $w_{y}$, integrating from $-x_{0}$ to $\infty$ and using (46) we get,

$$
\left.\left(R_{y} w_{y}-R w_{y y}\right)\right|_{-x_{0}} ^{\infty}+a \int_{-x_{0}}^{\infty}\left(y+x_{0}\right)^{q} w_{y} w^{p}=0 .
$$


Since $w$ decays exponentially as $|y| \rightarrow \infty$, we can replace $\int_{-x_{0}}^{\infty}$ by $\int_{-\infty}^{\infty}$. Using integration by parts we estimate

$$
\begin{aligned}
\int_{-x_{0}}^{\infty}\left(y+x_{0}\right)^{q} w_{y} w^{p} d y & \sim-\int_{-\infty}^{\infty} \frac{1}{p+1} w^{p+1} q\left(y+x_{0}\right)^{q-1} d y \\
& \sim-\frac{q}{p+1} x_{0}^{q-1} \int_{-\infty}^{\infty} w^{p+1} .
\end{aligned}
$$

Now for small $x$, we have we have that $R_{x x}-R \sim 0$ and $w \sim C_{0} e^{-\left|x-x_{0}\right|}$. The constant $C_{0}$ is obtained by expanding $w$ in the far-field $|y| \rightarrow \infty$. Thus we have

$$
w \sim C_{0} e^{-x_{0}} e^{x} ; \quad R \sim C_{1} e^{x}+C_{2} e^{-x} ; \quad x \sim 0 .
$$

Since $R$ must remain small as $x$ is increased, it follows that $C_{1}=0$. Moreover, $\left(R_{x}+U_{x}\right)_{x=0}=0$ which implies $C_{2}=C_{0} e^{-x_{0}}$. We therefore obtain

$$
\left(R_{y} w_{y}-R w_{y y}\right)_{y=-x_{0}}=2 C_{0}^{2} e^{-2 x_{0}} .
$$

This yields the following formula for $x_{0}$,

$$
2 C_{0}^{2} e^{-2 x_{0}} \sim a \frac{q}{p+1} x_{0}^{q-1} \int_{-\infty}^{\infty} w^{p+1} ; \quad a \ll 1, x_{0} \gg 1 .
$$

In case $p=2$, we have $w(y)=\frac{3}{2} \operatorname{sech}^{2}(y / 2)$; and $C_{0}=6 ; \int w^{3}=36 / 5$ so that

$$
\frac{e^{-2 x_{0}}}{x_{0}^{q-1}} \sim a \frac{q}{30} ; \quad p=2
$$

In case $p=3$ we have $U(y)=\sqrt{2} \operatorname{sech}(y)$ and $C_{0}=2 \sqrt{2} ; \quad \int w^{3}=\pi \sqrt{2} ;$ so that

$$
\frac{e^{-2 x_{0}}}{x_{0}^{q-1}} \sim a q \frac{\pi \sqrt{2}}{64} ; \quad p=3
$$

Ring solutions in higher dimension, generic case. We consider (4) with $N \geq 2$, in the limit $a \ll 1$. It is convenient to set

$$
\varepsilon:=a^{1 / q}
$$

so that (4) becomes

$$
0=u_{r r}+\frac{N-1}{r} u_{r}-u+u^{p}\left(1+(\varepsilon r)^{q}\right)
$$

The expansion we use is

$$
r=\frac{1}{\varepsilon} r_{0}+y ; \quad u=U_{0}(y)+\varepsilon U_{1}(y)+\cdots
$$

Expanding to two orders we obtain

$$
a r^{q}=\left(r_{0}+\varepsilon y\right)^{q}=r_{0}^{q}+\varepsilon q r_{0}^{q-1} y+\cdots
$$




$$
\begin{aligned}
& 0=U_{0 y y}-U_{0}+\left(1+r_{0}^{q}\right) U_{0}^{p} \\
& 0=U_{1 y y}-U_{1}+\frac{(N-1)}{r_{0}} U_{0 y}+r_{0}^{q} p U_{0}^{p-1}+U_{0}^{p} q r_{0}^{q-1} y
\end{aligned}
$$

Multiply (52) by $U_{0 y}$, and integrate by parts; using (51) we obtain

$$
\frac{q r_{0}^{q}}{(p+1)} \int_{-\infty}^{\infty} U_{0}^{p+1}=(N-1) \int_{-\infty}^{\infty} U_{0 y}^{2}
$$

The integrals can be further eliminated using Pohazhaev-type identities. Namely, multiply (51) by $U_{0}$ and integrate to get:

$$
-\int U_{0 y}^{2}-\int U_{0}^{2}+\left(1+r_{0}^{q}\right) \int U_{0}^{p+1}
$$

Multiply (51) by $y U_{0 y}$ and integrate to obtain

$$
-\frac{1}{2} \int U_{0 y}^{2}+\frac{1}{2} \int U_{0}^{2}-\left(1+r_{0}^{q}\right) \int \frac{U_{0}^{p+1}}{p+1}=0
$$

Combining (54) and (55) we obtain

$$
-2 \int U_{0 y}^{2}+\left(1+r_{0}^{q}\right) \int U_{0}^{p+1}\left(1-\frac{2}{p+1}\right)=0 .
$$

Substituting (56) into (53) and we finally obtain

$$
r_{0}^{q}=\frac{(N-1)(p-1)}{2 q-(N-1)(p-1)}
$$

In particular to solution to (57), ring solution exists if

$$
q>q_{c}=\frac{(N-1)(p-1)}{2} .
$$

This is consistent with thresholds derived in Theorem 1 for the case $N \geq 3$; in particular, it is in agreement with the bifurcation diagram shown on Figure 2(b): for $q>q_{c}$, the curve approaches $a \rightarrow 0$ as $s \rightarrow 0$.

Ring solutions in dimension $N=3$, threshold case $p=q+1$. The analysis of this case is very delicate and the asymptotics are very tricky. For simplicity, we consider only the case $p=2$. However the result generalizes without difficulty for any $p>1$. We summarize the result as follows.

Theorem 7. Suppose $N=3, p=2$ and $q=1$. In the limit $a \gg 1$, Let $r_{0} \gg 1$ be the large solution to the equation

$$
a=\frac{1}{30} r_{0}^{-2} \exp \left(2 r_{0}\right) ; \quad a, r_{0} \gg 1 .
$$

Then there exists solutions of (4) of the form

$$
u(r) \sim \frac{1}{r_{0} a} w\left(r-r_{0}\right)
$$


Proof of Theorem 7. We rescale

$$
u(r)=\frac{1}{r_{0} a} U(r)
$$

and define

so that

$$
\varepsilon=\frac{1}{a r_{0}}
$$

$$
0=U_{r r}+\frac{2}{r} U_{r}-U+U^{2}\left(\varepsilon+\frac{r}{r_{0}}\right) .
$$

The main idea is to separately solve the equation on $\left[0, r_{0}\right]$, then on $\left[r_{0}, \infty\right)$. Then $\varepsilon$ will be determined by requiring that $U\left(r_{0}^{-}\right)=U\left(r_{0}^{+}\right)$. So we treat (59) as two separate equations to solve: the first on $\left[0, r_{0}\right]$ with boundary conditions $U^{\prime}(0)=0=U^{\prime}\left(r_{0}\right)$ and the second on $\left[r_{0}, \infty\right)$ with boundary condtions $U^{\prime}\left(r_{0}\right)=0=U^{\prime}(\infty)$.

It will be shown below that $\varepsilon=O\left(r_{0} e^{-2 r_{0}}\right)$. Therefore we will need to expand in both $\varepsilon$ and $\frac{1}{r_{0}}$. First, we treat $r_{0}$ as constant with respect to $\varepsilon$ and expand

We get

$$
U=U_{0}+\varepsilon U_{1}+\ldots
$$

$$
\begin{aligned}
& 0=U_{0 r r}+\frac{2}{r} U_{0 r}-U_{0}+U_{0}^{2} \frac{r}{r_{0}} \\
& 0=U_{1 r r}+\frac{2}{r} U_{1 r}-U_{1}+2 U_{0} U_{1}+U_{0}^{2}
\end{aligned}
$$

Next we let

and expand

$$
y=r-r_{0}
$$

$$
U_{0}(r)=U_{00}(y)+\frac{1}{r_{0}} U_{01}(y)+\frac{1}{r_{0}^{2}} U_{02}(y)+\ldots
$$

We have

$$
\left(U_{00}\right)_{y y}-U_{0}+U_{0}^{2}=0 ; \quad U_{00}^{\prime}(0)=0
$$

so that

At the next order we get

$$
U_{00}(y)=w(y)
$$

$$
L U_{01}+2 w_{y}+y w^{2}=0
$$

where

$$
L \phi:=\phi_{y y}-\phi+2 w \phi .
$$

Note that $L(y w)=y w^{2}+2 w_{y}$ so that solution to (60) is given by

$$
U_{01}=-y w+C w_{y} .
$$

To determine the constant $C$ we impose the condition $U_{00}^{\prime}(0)=0$ which yields $C=-2$,

$$
U_{01}=-y w^{2}-2 w_{y} .
$$


Therefore $U_{01}$ is odd and at the next order we get

$$
L U_{02}=f(y)
$$

where $f(y)$ is a purely even function. Again, we treat this as two equations, one to the left and another to the right of $r_{0}$. To the left of $r_{0}$, multiply (61) by $w_{y}$ and integrate $y=-r_{0} \ldots 0$. We then get

$$
\left(w_{y} U_{02 y}-w_{y y} U_{02}\right)_{y=-r_{0}}^{y=0^{-}} \sim \int_{-\infty}^{0} f(y) w_{y} d y=-\int_{0}^{\infty} f(y) w_{y} d y .
$$

To the right of $r_{0}$ we get

$$
\left(w_{y} U_{02 y}-w_{y y} U_{02}\right)_{y=0^{+}}^{y=\infty} \sim \int_{0}^{\infty} f(y) w_{y} d y .
$$

Adding the equations (62) and (63) together we get

$$
w_{y y}(0)\left[U_{02}\left(0^{+}\right)-U_{02}\left(0^{-}\right)\right]=\left(w_{y} U_{02 y}-w_{y y} U_{02}\right)_{y=-r_{0}} .
$$

Therefore we need to determine the behaviour near $r=0$. Recalling that $y=r+r_{0}$ we write

$$
w \sim C_{0} e^{r}, \quad r \sim 0 ; \quad C_{0}=6 e^{-r_{0}} .
$$

Since the solution decays near zero, we have $u^{2} \ll u$ so that for small $r$

$$
u_{r r}+\frac{2}{r} u_{r}-u \sim 0, u^{\prime}(0)=0 .
$$

Such solution is given by

$$
u=A \frac{e^{r}-e^{-r}}{r}
$$

where the constant $A$ is to be determined. To do so, we rewrite $U_{00}+\frac{1}{r_{0}} U_{01}$ as

$$
\begin{aligned}
U_{00}+\frac{1}{r_{0}} U_{01} & \sim w+\frac{1}{r_{0}}\left(-2 w_{y}-y w\right) \\
& \sim w+\frac{1}{r}(-2-y w) \\
& \sim C_{0} e^{r}\left(1+\frac{1}{r}\left(-2-\left(r-r_{0}\right)\right)\right. \\
& \sim C_{0} \frac{e^{r}}{r}\left(r_{0}-2\right) .
\end{aligned}
$$

We now match this with the growing mode of (64) to obtain

$$
A=C_{0}\left(r_{0}-2\right)
$$

Therefore the uniform expansion of $u$ is given by

$$
u \sim w+\frac{1}{r}(-2 w-y w)-C_{0}\left(r_{0}-2\right) \frac{e^{-r}}{r} .
$$


We now match decaying mode of (64) to the remainder of $U_{0}$ in the outer region:

$$
-A \frac{e^{-r}}{r} \sim \frac{U_{02}}{r_{0}^{2}} \sim \frac{1}{r} \frac{U_{02}}{r_{0}}
$$

This gives the following behaviour of $U_{02}$ in the outer region:

$$
U_{02} \sim r_{0} C_{0}\left(2-r_{0}\right) e^{-r}, \quad r \rightarrow 0 .
$$

Using this we evaluate

$$
\left(w_{y} U_{02 y}-w_{y y} U_{02}\right)_{y=-r_{0}} \sim 2 C_{0}^{2} r_{0}\left(r_{0}-2\right)
$$

Recalling that $w_{y y}(0)=-\frac{3}{4}$ we get

$$
U_{02}\left(0^{+}\right)-U_{02}\left(0^{-}\right)=-\frac{8}{3} C_{0}^{2} r_{0}^{2}\left(1-\frac{2}{r_{0}}\right)
$$

This yields

$$
U_{0}\left(0^{+}\right)-U_{0}\left(0^{-}\right) \sim-\frac{8}{3} 36 e^{-2 r_{0}}\left(1-\frac{1}{r_{0}}\right) .
$$

Next we compute the jump in $U_{1}$. We expand

$$
U_{1}=U_{10}(y)+\frac{1}{r_{0}} U_{11}(y)+\ldots
$$

The leading order is

$$
L U_{10}+w^{2}=0 .
$$

Imposing $U_{10}^{\prime}(0)=0$ and recalling that $L w=w^{2}$, we get

$$
U_{10}(y)=-w \text {. }
$$

The next order then becomes

$$
L U_{11}=2 w_{y}+2 y w^{2} .
$$

Multiplying by $w_{y}$ and integrating to the left of $r_{0}$ we therefore get

$$
\left(w_{y} U_{11 y}-w_{y y} U_{11}\right)_{y=-r_{0}}^{0^{-}}=\int_{-\infty}^{0}\left(2 w_{y}+2 y w^{2}\right) w_{y}=-\frac{6}{5}
$$

and similarly to the right of $r_{0}$,

$$
\left(w_{y} U_{11 y}-w_{y y} U_{11}\right)_{0^{+}}^{\infty}=\int_{0}^{\infty}\left(2 w_{y}+2 y w^{2}\right) w_{y}=-\frac{6}{5}
$$

Adding $(72,73)$ together and ignoring the exponentially small boundary terms we obtain

$$
U_{11}\left(0^{+}\right)-U_{11}\left(0^{-}\right)=\frac{16}{5}
$$

so that

$$
U_{1}\left(r_{0}^{+}\right)-U_{1}\left(r_{0}^{-}\right)=\frac{16}{5 r_{0}}
$$


Putting together (70) and (74) we have

$$
\begin{aligned}
u\left(r_{0}^{+}\right)-u\left(r_{0}^{-}\right) & \sim\left(U_{1}\left(r_{0}^{+}\right)-U_{1}\left(r_{0}^{-}\right)\right)+\varepsilon\left(U_{1}\left(r_{0}^{+}\right)-U_{1}\left(r_{0}^{-}\right)\right) \\
& \sim-\frac{8}{3} 36 e^{-2 r_{0}}\left(1-\frac{1}{r_{0}}\right)+\frac{\varepsilon}{r_{0}} \frac{16}{5}
\end{aligned}
$$

The solvability condition is that this quantity is zero, that is

$$
\varepsilon \sim 30 r_{0} e^{-2 r_{0}}\left(1-\frac{1}{r_{0}}\right) \text {. }
$$

This completes the proof.

Appendix C. Phase Plane Analysis of $u^{\prime \prime}+\frac{N-1}{r} u^{\prime}+u^{p} r^{q}=0 ; \quad u(0)=1, u^{\prime}(0)=0$.

The ODE in the title can be written as

$$
\left(r^{N-1} u^{\prime}\right)^{\prime}+r^{q+N-1} u^{p}=0 ; \quad u(0)=1, u^{\prime}(0)=0 .
$$

In fact this equation is a special case of the ODE studied in the classical paper [10], where the scaling symmetry of (75) is utilized to enable a complete qualitative analysis of (75) and related equations. For completeness, we provide a short derivation of this analysis as applied to (75) in this appendix. The main result that we need is the following.

Lemma 8. Suppose that $p>1, q>q_{\star}$, where $q_{\star}$ is given in (7a). Then the solution to (75) crosses the horizontal axis.

Proof. Make a change of variables

$$
r=e^{s} ; \quad u(r)=e^{-b s} v(s) .
$$

where

$$
b=\frac{q+2}{p-1}
$$

Then (75) becomes

$$
v^{\prime \prime}+(2 \alpha+n-2) v^{\prime}+\alpha v+v^{p}=0 .
$$

Letting $w=v^{\prime},(75)$ is reduced to an autonomous system

$$
\left\{\begin{array}{c}
v^{\prime}=w \\
w^{\prime}=a w+b v-v^{p}
\end{array}\right.
$$

where

$$
a:=\frac{2+2 q+2 p-n(p-1)}{p-1} ; \quad b:=\frac{q+2}{p-1} .
$$




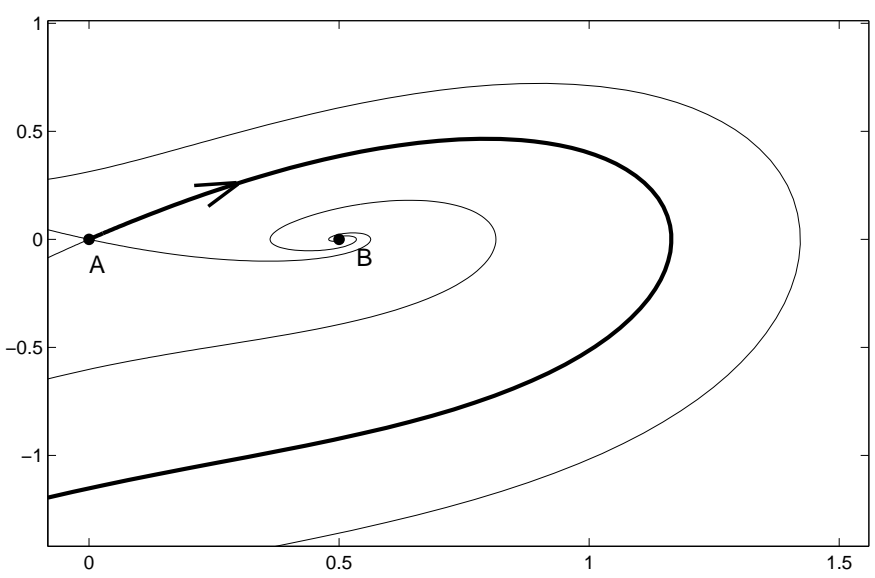

Figure 4. Phase portrait of (79) with $p=2, a=0.5, b=0.5$. The thick curve indicates the unstable manifold out of $A$; it eventually intersects the $v$-axis.

Now the system (79) has two equilibria points, $A: v, w=0$ and $B: v=b^{1 /(p-1)}, w=0$. Their eigenvalues are:

$$
\begin{aligned}
& A: \lambda=\frac{a \pm \sqrt{a^{2}-4 b}}{2} \\
& B: \lambda=\frac{a \pm \sqrt{a^{2}+4 b(p-1)}}{2}
\end{aligned}
$$

Note that $q=q_{\star} \Longleftrightarrow a=0$ and hence $a>0 ; b>0$. From linearization it then follows that $A$ is a saddle point and $B$ is an unstable equilibrium. Moreover the initial conditon $u^{\prime}(0)=0$ corresponds to

$$
w / v \sim b \text { as } s \rightarrow-\infty
$$

the condition $u(0)=1$ implies that $v \rightarrow 0$ as $s \rightarrow-\infty$. Thus the solution to (75) lies on the unstable manifold coming out of the saddle point at the origin. From phase plane analysis, such solution will cross the $v$ axis for some $s$ (see Figure 4). Hence $u$ will cross the horizontal axis for some $r>0$.

\section{ACKNOWLEDGEMENTS}

TK is supported by an NSERC discovery grant, Canada. TK would also like to thank the Department of Mathematics, National Taiwan University for their kind hospitality, where a part of this paper was written.

\section{REFERENCES}

[1] W-Y. Ding and W-M. Ni, "On the Existence of Positive Entire Solutions of a Semilinear Elliptic Equation", Archive for Rational Mechanics and Analysis, Vol. 91, No. 1 (Dec, 1986), pp. 283-308 
[2] A. Doelman, R. A. Gardner, T. J. Kaper, Stability Analysis of Singular Patterns in the 1D Gray-Scott Model: A Matched Asymptotics Approach, Physica D, 122, No. 1-4, (1998), pp. 1-36.

[3] A. Doelman, T. J. Kaper, P. Zegeling, Pattern Formation in the One-Dimensional Gray-Scott Model, Nonlinearity, 10, No. 2, (1997), pp. 523-563.

[4] A. Doelman, T. J. Kaper, L. A. Peletier, Homoclinic Bifurcations at the Onset of Pulse Replication, J. Differential Equations, 231 (2006) pp. 359-423.

[5] S. Ei, Y. Nishiura, K. Ueda, $2^{n}$ Splitting or Edge Splitting?: A Manner of Splitting in Dissipative Systems, Japan. J. Indust. Appl. Math., 18, No. 2, (2001), pp. 181-205.

[6] Y. Hayase, T. Ohta, Sierpinski Gasket in a Reaction-Diffusion System, Phys. Rev. Lett., 81, No. 8, (1998), pp. $1726-1729$

[7] Y. Hayase, Sierpinski Gaskets in Excitable Media, Phys. Rev. E, 62, No. 5, (2000), pp. 5998-6003.

[8] Y. Hayase, T. Ohta, Self-Replication of a Pulse in Excitable Reaction-Diffusion Systems, Phys. Rev. E., 66, No. 3, (2002), 036218.

[9] A. Doelman, H. van der Ploeg, Homoclinic Stripe Patterns, SIAM J. Appl. Dyn. Systems, 1, No. 1, (2002), pp. $65-104$.

[10] D.D. Joseph and T.S. Lundgren, Quazilinear Dirichlet problems driven by positive sources, Arch.Rat.Mech.Anal., Vol. 49.

[11] K. J. Lee, H. L. Swinney, Lamellar Structures and Self-Replicating Spots in a Reaction-Diffusion System, Phys. Rev. E., 51, No. 3, (1995), pp. 1899-1915.

[12] T. Kolokolnikov and M. Tlidi, Spot deformation and replication in the two-dimensional Belousov-Zhabotinski reaction in water-in-oil microemulsion, Physical Review Letters, Vol.98 2007(18), article 188303.

[13] T. Kolokolnikov, M. J. Ward, J. Wei, Self-replication of mesa patterns in reaction-diffusion models, Physica D, Vol.236(2), 2007, Pages 104-122

[14] T. Kolokolnikov, M. J. Ward, J. Wei, The Existence and Stability of Spike Equilibria in the One-Dimensional Gray-Scott Model: The Pulse-Splitting Regime, Physica D, 202, No. 3-4, (2005), pp. 258-293.

[15] T. Kolokolnikov, M. J. Ward, J. Wei, The Stability of a Stripe for the Gierer-Meinhardt Model and the Effect of Saturation, SIAM J. Appl. Dyn. Sys., 5, No. 2, (2006), pp. 313-363.

[16] H. Meinhardt, The Algorithmic Beauty of Sea Shells, Springer-Verlag, Berlin (1995).

[17] Man Kam Kwong and Yi Li, "Uniqueness of Radial Solutions of Semilinear Elliptic Equations", Transactions of the American Mathematical Society, Vol. 333, No. 1 (Sep., 1992), pp. 339-363

[18] A. P. Muñuzuri, V. Pérez-Villar, M. Markus, Splitting of Autowaves in an Active Medium, Phys. Rev. Lett., 79, No. 10, (1997), pp. 1941-1945.

[19] C. Muratov, V. V. Osipov, Static Spike Autosolitons in the Gray-Scott Model, J. Phys. A: Math Gen. 33, (2000), pp. 8893-8916.

[20] C. Muratov, V. V. Osipov, Stability of the static spike autosolitons in the Gray-Scott model, SIAM J.Appl. Math, Vol. 62, No. 5, pp. 1463-1487

[21] Y. Nishiura, D. Ueyama, A Skeleton Structure of Self-Replicating Dynamics, Physica D, 130, No. 1, (1999), pp. 73-104.

[22] Y. Nishiura, D. Ueyama, Spatio-Temporal Chaos for the Gray-Scott Model, Physica D, 150, No. 3-4, (2001), pp. 137-162.

[23] J. E. Pearson, Complex Patterns in a Simple System, Science, 216, (1993), pp. 189-192.

[24] W. N. Reynolds, S. Ponce-Dawson, J. E. Pearson, Dynamics of Self-Replicating Patterns in Reaction-Diffusion Systems, Phys. Rev. Lett., 72, No. 17, (1994), pp. 2797-2800.

[25] W. N. Reynolds, S. Ponce-Dawson, J. E. Pearson, Dynamics of Self-Replicating Spots in Reaction-Diffusion Systems, Phys. Rev. E, 56, No. 1, (1997), pp. 185-198.

[26] Walter A. Strauss, "Partial differential equations, an introduction", John Wiley \& Sons Inc, 1992.

[27] J. Wei, Existence and Stability of Spikes for the Gierer-Meinhardt System, Handbook of differential equations, stationary partial differential equations, volume 5 (M. Chipot ed.), Elservier. pp. 489-581. 
[28] J. Wei, "On single interior spike solutions of Gierer-Meinhardt system: uniqueness and spectrum estimates" Eur. J. Appl. Math. 10 (1999), 353-378. 